1 Beverage carbohydrate concentration influences the intermittent endurance capacity of adolescent team games players during prolonged intermittent running.

5 Authors:

6 Shaun M. Phillips, Anthony P. Turner, Mark F. Sanderson \& John Sproule.

7

\title{
8 Affiliation:
}

9 Institute of Sport, Physical Education and Health Sciences,

10 University of Edinburgh,

11 Edinburgh,

12 EH8 8AQ, UK.

14 Correspondence:

15 Mr Shaun Phillips

16 Institute of Sport, Physical Education and Health Sciences

17 University of Edinburgh

18 St Leonards Land

19 Holyrood Road

20 Edinburgh

21 EH8 8AQ

22 UK

$23+44(0) 1316509788$

$24 \quad$ S.M.Phillips@sms.ed.ac.uk 


\section{Abstract}

3 This study investigated the influence of consuming a 2, 6, and $10 \%$ carbohydrate-electrolyte

4 (CHO-E) solution on the intermittent endurance capacity and sprint performance of

5 adolescent team games players. Seven participants (five males and two females; mean age

$6 \quad 13.3 \pm 0.5$ years, height $1.71 \pm 0.05 \mathrm{~m}$, body mass $(\mathrm{BM}) 62.0 \pm 6.3 \mathrm{~kg}$ ) performed three trials

7 separated by 3 to 7 days. In each trial, they completed four 15 min periods of part $A$ of the

8 Loughborough Intermittent Shuttle Test (LIST) followed by an intermittent run to exhaustion

9 (part B). Participants consumed $5 \mathrm{ml} . \mathrm{kg}^{-1} \mathrm{BM}$ of the solution during the 5 min pre-exercise

10 period, and a further $2 \mathrm{ml} . \mathrm{kg}^{-1} \mathrm{BM}$ every 15 min during part A of the LIST. Intermittent

11 endurance capacity increased by $34 \%$ with ingestion of the $6 \%$ CHO-E solution compared

12 with the $10 \%$ solution $(5.5 \pm 0.8$ vs. $4.1 \pm 1.5 \mathrm{~min}, P<0.05)$, equating to a distance of $931 \pm$

13172 vs. $706 \pm 272 \mathrm{~m}(P<0.05)$. There was no significant difference between the $2 \%(4.8 \pm$

$141.2 \mathrm{~min})$ and $6 \%(P=0.10)$ or the $2 \%$ and $10 \%$ solutions $(P=0.09)$. Carbohydrate

15 concentration did not significantly influence mean $15 \mathrm{~m}$ sprint time $(P=0.38)$. These results

16 suggest that the carbohydrate concentration of an ingested solution influences the

17 intermittent endurance capacity of adolescent team games players with a $6 \%$ solution

18 significantly more effective than a $10 \%$ solution.

24 Key Words: Team games; performance; nutrition; young people; LIST 


\section{Introduction}

3 We recently reported a significant $24 \%$ improvement in intermittent endurance running

4 capacity (hereafter referred to as intermittent endurance capacity) when 12-14 year old team

5 games athletes ingested a $6 \%$ carbohydrate-electrolyte (CHO-E) solution before and during a

6 modified Loughborough Intermittent Shuttle Test (LIST, Phillips et al 2010). This was

7 achieved using the same body mass (BM)-standardised ingestion volumes and timings as the

8 original adult work of Nicholas et al (1995). The lack of a significant treatment effect on

9 heart rate (HR), ratings of perceived exertion (RPE), sweat rate (SR) or BM loss in our

10 previous study also mirrored the findings of most relevant adult studies (Ali et al 2007; Davis

11 et al 1999; Nicholas et al 1995; Welsh et al 2002). This suggests that the relative

12 physiological responses to intermittent endurance running with carbohydrate (CHO)

13 supplementation appear similar between adolescents and adults.

15 Most previous adult studies that demonstrated improved intermittent endurance capacity with $16 \mathrm{CHO}$ ingestion during intermittent endurance running used a $\mathrm{CHO}$ concentration ([CHO]) of $17 \quad 6.0-6.9 \%\left(60-69\right.$ g.L $L^{-1}$ of solution, Davis et al 1999; Nicholas et al 1995; Welsh et al 2002).

18 This is similar to existing guidelines for $\mathrm{CHO}$ supplementation during prolonged steady-state 19 exercise for adults, i.e. a recommendation of $\sim 1.0-1.1 \mathrm{~g} \cdot \mathrm{min}^{-1}\left(60-70 \mathrm{~g} \cdot \mathrm{h}^{-1}\right.$, Jeukendrup 2004)

20 to maximise exogenous $\mathrm{CHO}\left(\mathrm{CHO}_{\text {exo }}\right)$ oxidation. However, it cannot be assumed that these 21 guidelines for steady-state exercise also apply to $\mathrm{CHO}$ ingestion during participation in team games. Christmass et al (1999) demonstrated a 1.2 times higher $(P<0.05)$ rate of endogenous $\mathrm{CHO}\left(\mathrm{CHO}_{\text {endo }}\right)$ oxidation during 90 min of sustained intermittent compared with

24 continuous running at the same overall $\dot{\mathrm{VO}}_{2}$. This suggests that the requirement for $\mathrm{CHO}$ may be greater during intermittent compared with continuous exercise. Some studies have 
1 investigated the influence of $[\mathrm{CHO}]$ on endurance capacity during prolonged intermittent

2 exercise (Murray et al., 1987). However, protocol issues make drawing conclusions on the

3 influence of $[\mathrm{CHO}]$ difficult, and also preclude the application of the findings to team games.

4 To date, no published research has investigated the influence of consuming different [CHO]

5 during intermittent endurance running. Ali and Williams (2009) reported no benefit of

6 ingesting $\mathrm{CHO}$ at a rate of $52 \mathrm{~g} \cdot \mathrm{h}^{-1}$ on sprint performance during the LIST, but did report a

7 significant improvement in sprint performance with ingestion of $32 \mathrm{~g} \mathrm{CHO}^{-1}$ (Ali et al

8 2007). However, intermittent endurance capacity, where CHO ingestion most consistently

9 exerts an effect during intermittent endurance running, was not assessed in these studies.

11 Due to a lack of empirical research, no published guidelines exist for $\mathrm{CHO}$ supplementation during team games exercise in adolescents. The findings of Phillips et al (2010) were generated despite one fewer drink period compared with adult work, as adolescents commonly play team games for a shorter duration than adults (60 min vs. 90 min, Ekblom 1986). As a result, mean CHO intake was 0.56 g. $\mathrm{min}^{-1}$ compared with $~ 0.79-1.3$ g. $\mathrm{min}^{-1}$ in adult work (Nicholas et al 1995; Welsh et al 2002). While the shorter duration of adolescent team games may suggest a lesser depletion of $\mathrm{CHO}_{\text {endo }}$ stores, and therefore question the efficacy of $\mathrm{CHO}$ supplementation, it should be considered that adolescents may have lower endogenous glycogen stores than adults (Aucouturier et al 2008), which may offset the

20 sparing effect of a shorter exercise bout. Furthermore, $\mathrm{BM}$-relative $\mathrm{CHO}_{\text {exo }}$ oxidation rates 21 may be significantly greater in young people compared to adults (Timmons et al 2003), 22 despite the preferential use of fat as a fuel source in young people (Timmons et al 2007).

23 This is likely a mechanism to preserve the lower $\mathrm{CHO}_{\text {endo }}$ stores (Riddell 2008). The

24 different metabolic response of young people to exercise indicates that adult guidelines

25 regarding $\mathrm{CHO}$ supplementation before and during exercise may not be appropriate for this 
1 population. It would be of interest to study the influence of different rates of CHO ingestion

2 by young people during intermittent endurance running. This would enable observation of

3 whether their ability to readily oxidise $\mathrm{CHO}_{\text {exo }}$ elicits a dose-response relationship to $\mathrm{CHO}$

4 provision in terms of enhancing exercise performance (Jeukendrup et al 1999), and to begin

5 the process of forming guidelines for the ingestion of $\mathrm{CHO}$ during intermittent endurance

6 running in this population.

7

8 Manipulating $\mathrm{CHO}_{\text {exo }}$ intake could be achieved by ingesting different volumes of a $6 \%$

9 solution; however, ingesting larger volumes may lead to gastrointestinal distress (Shi et al

10 2004). Furthermore, this practice would not translate well to actual field-based team games,

11 where there are limited opportunities to drink during matches (Clarke et al 2008).

12 Manipulating the $[\mathrm{CHO}]$ of the ingested solution may also increase the risk of gastrointestinal

13 distress (Shi et al 2004), but the minimal understanding of $\mathrm{CHO}$ tolerance during team games

14 in adolescents, along with the absence of any $\mathrm{CHO}$ intake guidelines, provides a rationale for

15 using different [CHO].

17 The aim of this study is to determine the influence of ingesting a 2, 6, and $10 \%$ CHO-E

18 solution immediately before, and during, an intermittent endurance running protocol on the

19 intermittent endurance capacity and sprint performance of adolescent team games players. It

20 was hypothesised that [CHO] would significantly influence intermittent endurance capacity,

21 but would not significantly impact sprint performance during prolonged intermittent running. 


\section{$1 \quad$ Methods}

Participants

5 Seven team games players (five males and two females; mean age $13.3 \pm 0.5$ years, height

$6 \quad 1.71 \pm 0.05 \mathrm{~m}, \mathrm{BM} 62.0 \pm 6.3 \mathrm{~kg}$ ) participated in the study. Participants were recruited from

7 local schools and sports clubs. Inclusion criteria were that they had to be between the ages of

$8 \quad 12-14$ years, regularly participating in competitive soccer, rugby or field hockey to at least

9 club level, free from any muscle or joint injury, and not taking medication that influences the

10 ability to exercise. All participants were in good health at the time of the study, as

11 determined by completion of a pre-study medical questionnaire. Participants' were either

12 frequent or occasional users of $\mathrm{CHO}$ containing sports drinks.

14 Prior to inclusion, comprehensive written and verbal explanation of the study was given to

15 participants and parents, and written parental informed consent was received. Each

16 participant then gave their written assent. The study received ethical approval from the

17 University of Edinburgh Ethics Committee.

Biological maturity status

21 Biological maturity was not assessed using direct observational assessment of Tanner Stages

22 (Tanner 1962), due to ethical and consensual restrictions. Instead, biological maturity offset was assessed using the established, non-invasive equations of Mirwald et al (2002), as

24 previously described (Phillips et al 2010). For the participants in this study, mean biological maturity offset was +1.25 years (range +0.70 to +2.68 years). Mean predicted age at peak 
1 height velocity for females was 11.3 years (range 0 years) and for males was 13.0 years

2 (range 12.3 to 13.8 years). This classifies the participants in this study as average maturers

3 (Baxter-Jones et al 2005).

4

5 Preliminary Tests

6

$7 \quad$ Peak Running Velocity

8

9 All exercise intensities used in the main experimental protocol were based on percentages of 10 peak running velocity $\left(\mathrm{V}_{\text {peak }}\right)$ as determined from a treadmill $\mathrm{V}_{\text {peak }}$ test. This is in contrast to 11 the more common calculation of speed based on percentage of $\dot{\mathrm{V}} \mathrm{O}_{2 \mathrm{max}}$, and is believed to 12 more accurately reflect physiological demand during team games (Bangsbo 1994). The 13 physiological responses to incremental maximal treadmill running and free-range running 14 have been reported to be similar (Crouter et al., 2001). Prior to undertaking the $\mathrm{V}_{\text {peak }}$ test, all 15 participants walked at a self-selected speed on the treadmill (Ergo 55, Woodway, Germany)

16 for $2 \mathrm{~min}$, then completed the first four levels of the $\mathrm{V}_{\text {peak }}$ test as described below, to

17 familiarise themselves with the treadmill (Lavcanska et al 2005). This also acted as a

18 standardised warm-up. Following this familiarisation, participants sat quietly for 10 min to

19 recover and allow any excessive anxiety to dissipate before starting the test.

21 The $\mathrm{V}_{\text {peak }}$ test, adapted from Marino et al (2004), began at $8 \mathrm{~km} \cdot \mathrm{h}^{-1}$ at a gradient of $1 \%$ for 22 one-minute, after which the speed was increased by $0.5 \mathrm{~km} \cdot \mathrm{h}^{-1}$ in one-minute increments until 23 the participant indicated they could not continue, despite strong verbal encouragement. A 24 maximal effort was confirmed by observation of subjective symptoms of fatigue (facial 25 flushing, unsteady gait, heavy sweating, hyperpnoea) and attainment of a HR $\geq 195$ beats per 
1 min (Armstrong 2007). Peak running velocity and maximum $H R\left(H_{\max }\right)$ were calculated as

2 the highest treadmill velocity maintained for $30 \mathrm{~s}$ and the highest $5 \mathrm{~s}$ average, respectively.

3 After a 15 min seated recovery, participants performed $15 \mathrm{~min}$ of the LIST, as described

4 below, to familiarise themselves with the running speeds required and the data collection

5 procedures.

6

$7 \quad$ Experimental Design

8

9 All participants completed three trials separated by a minimum of three, and maximum of

10 seven, days, in a randomised, counterbalanced, double-blind fashion. The three trials were as 11 follows:

12

13 A. $2 \%$ CHO-E solution (low $\mathrm{CHO}$ trial - LCHO)

14 B. $6 \% \mathrm{CHO}-\mathrm{E}$ solution (moderate $\mathrm{CHO}$ trial - $\mathrm{MCHO}$ )

15 C. $10 \%$ CHO-E solution (High $\mathrm{CHO}$ trial $-\mathrm{HCHO}$ )

17 The $[\mathrm{CHO}]$ of both the $\mathrm{LCHO}$ and $\mathrm{MCHO}$ solutions was similar to that of commercially available $\mathrm{CHO}-\mathrm{E}$ drinks. Furthermore, the $[\mathrm{CHO}]$ of the $\mathrm{MCHO}$ solution was the same as that used in previous research from this laboratory (Phillips et al 2010) and was similar to

20 solutions used in the majority of adult work (Davis et al 1999; Nicholas et al 1995; Welsh et 21 al 2002). The HCHO solution was employed, as solutions with a [CHO] $>10 \%$ are rarely used in contemporary research due to current adult guidelines regarding fluid and $\mathrm{CHO}$

23 intake during prolonged, steady-state exercise (Jeukendrup 2004). No such guidelines

24 currently exist for young people. Therefore, the use of a $[\mathrm{CHO}]$ greater than $10 \%$ currently 
1 has no empirical support and, due to the lack of knowledge of $\mathrm{CHO}$ tolerance during

2 prolonged intermittent exercise in young people, no ethical basis.

4 The $\mathrm{CHO}$ was $100 \%$ maltodextrin (High5 Ltd, Bardon, UK). Commercially available 5 electrolyte tablets (High5 Ltd, Bardon, UK) were used in all solutions (one tablet dissolved

6 per $500 \mathrm{ml}$ of solution), yielding the following electrolyte concentrations per L: sodium, 250

7 mg; magnesium, $60 \mathrm{mg}$; potassium, $90 \mathrm{mg}$; calcium, $20 \mathrm{mg}$. The electrolyte tablets also

8 contained a flavouring (citrus, berry, or cherry-orange). Prior to the first trial, each

9 participant was asked which flavour they would prefer. The participants' chosen flavour was

10 then used for all three of their trials. Therefore, within-participants, all solutions were

11 matched for colour, taste, texture, and feeling within the mouth. Participants were requested

12 to refrain from heavy physical activity for $48 \mathrm{~h}$ before each trial. They were also asked to

13 record their food and fluid intake, including the portion size of all food consumed and the

14 volume of all fluid ingested, for $24 \mathrm{~h}$ before the first trial. This diet was replicated prior to

15 trials two and three to standardise muscle and hepatic glycogen concentrations and hydration

16 status. Participants were not requested to record food and fluid intake in the depth of detail

17 that would have enabled a subsequent dietary composition analysis. Requesting this would

18 have placed greater stress on extremely time-pressured participants and their parents, and

19 may have negatively affected adherence to the dietary record, and retention of participants

20 through the full study.

Experimental Protocol

24 Standing height was measured using a free-standing adjustable stadiometer (Seca, model no.

25 2251821009, Germany). After voiding and urinating, if necessary, dry nude BM was 
1 recorded (Seca Digital, model no. 7052321009, Germany). Participants were then fitted with

2 a HR monitor chest strap and watch (Polar RS400, Polar Electro Oy, Finland) and sat quietly

3 for 5 min, after which a standardised warm-up consisting of jogging, striding and dynamic

4 stretching was undertaken for $10 \mathrm{~min}$. Immediately following the warm-up, participants sat

5 and were instructed to consume the prescribed solution $\left(5 \mathrm{ml} . \mathrm{kg}^{-1} \mathrm{BM}\right)$ during the $5 \mathrm{~min}$

6 before commencing exercise (Nicholas et al 1995). Once this initial bolus had been

7 consumed, participants were asked to state which solution they believed was being

8 prescribed.

10 The LIST was conducted indoors, on a level rubber floor, as described elsewhere (Phillips et 11 al 2010). Briefly, participants completed four blocks of part A of the LIST separated by 3

12 min seated recovery, followed by an intermittent run to exhaustion (part B). Participants

13 consumed the solution $\left(2 \mathrm{ml} \cdot \mathrm{kg}^{-1} \mathrm{BM}\right)$ in the recovery period between each 15 min block and

14 in the recovery period before commencing part B. After the measurement of post-exercise

15 BM, participants were asked again to state which solution they believed they had received

16 during the protocol. This was done in order to compare their response with their pre-exercise

17 choice, and observe whether their experiences during the exercise bout prompted them to

18 change their mind about which solution they had consumed during the protocol. Participants'

19 were clearly informed that they were free to change their mind from their pre-exercise

20 solution choice, or to keep their selection the same.

Measurements

24 Heart rate was recorded at $5 \mathrm{~s}$ intervals throughout the $\mathrm{V}_{\text {peak }}$ test and the experimental 25 protocol using short-range telemetry. Data was retrieved and downloaded onto a computer 
1 software program (Polar ProTrainer 5, Polar Electro Oy, Finland) for subsequent analysis.

2 Ambient temperature and relative humidity were recorded immediately before the start of the

3 protocol and at the end of each 15 min block in part A using a digital hygro-thermometer

4 (Tako Astatic Technology, Malaysia). Ratings of perceived exertion were recorded during

5 the first shuttle of the final walking phase of each 15 min block in part A and at exhaustion in

6 part B using the Children's Omnibus Scale of Perceived Exertion (0-10 scale). This scale has

7 been validated for use with participants of the age range in this study (Roemmich et al 2006).

8 Gut fullness (GF) and gastric discomfort (GD) were assessed immediately on completion of

9 each 15 min block in part A and at exhaustion in part B via anchored 10 point scales $(1=$ not

10 at all, $10=$ extremely; van Nieuwenhoven et al 2005). Sprint times were measured in one

11 direction by two wireless infrared single-beam photoelectric cells (Speed Trap 2, Gill

12 Athletics, Illinois) placed $15 \mathrm{~m}$ apart. If participants needed to urinate at any time from the

13 onset of the protocol until completion of the measurement of post-exercise BM, they did so

14 into a measuring jug, with this volume incorporated into the BM loss calculation. No

15 participant needed to urinate during the protocol in the current study. Body mass loss was

16 calculated from the difference between pre- and post-exercise nude BM, corrected for fluid

17 intake and urine output. Sweat rate $\left(\mathrm{L}^{\mathrm{h}} \mathrm{h}^{-1}\right)$ was calculated using the equation: (Pre-exercise

$18 \mathrm{BM}(\mathrm{kg})+$ fluid ingested $(\mathrm{L})$ - urine output $(\mathrm{L})$ - post-exercise $\mathrm{BM}(\mathrm{kg}))$ / protocol duration

19 (min) x 60 (Edwards et al 2007). This calculation does not account for BM loss due to fuel

20 oxidation and respiratory fluid loss, but it is unlikely these would differ between trials

21 (Edwards et al 2007). 
3 The Shapiro-Wilk test for normality was employed on all data sets. One-way repeated measures ANOVA compared between trials differences in fluid and CHO intake, pre-exercise BM, BM loss and SR, and time to exhaustion, HR, RPE, GF and GD at exhaustion in part B.

6 Bonferroni pairwise comparisons and simple contrast analysis were used to explore main

7 effects of fluid and $\mathrm{CHO}$ intake and time to exhaustion, respectively. Two way (solution $\mathrm{x}$

8 time) ANOVA analysed mean relative humidity, mean sprint times and mean peak sprint

9 times, HR, RPE, GF and GD during part A. Bonferroni pairwise comparisons explored the

10 main effect for RPE, and paired t-tests with Bonferroni correction explored main effects for

11 mean sprint times, mean peak sprint times, HR, GF and GD. Friedman tests with Bonferroni

12 correction analysed between trials differences in mean ambient temperature at all time points,

13 with a Friedman's test employed to analyse the main effect of time for the grouped trials data.

14 Chi-square analysis assessed the frequency distribution of solution choice responses. Effect

15 sizes (ES) were calculated using partial eta squared $\left(\eta^{2}\right)$ values, which were square rooted to 16 give correlation coefficients (Field 2005). Where post-hoc paired t-tests with Bonferroni 17 correction were performed, ES was calculated using the equation of Rosnow and Rosenthal 18 (2005) to produce correlation coefficients. Effect sizes were defined as small $(r=0.1-0.3)$, moderate $(r=0.3-0.5)$, large $(r=0.5-0.7)$, very large $(r=0.7-0.9)$, and nearly perfect $(r=$

20 0.9-1.0) based on the classifications of Hopkins (2006). Data are mean \pm SD. With the 21 exception of analyses using the Bonferroni correction, significance was set at $P<0.05$. 


\section{$1 \quad$ Results}

5 Mean $\mathrm{V}_{\text {peak }}$ attained in the incremental treadmill run to exhaustion was $14.4 \pm 1.2 \mathrm{~km} \cdot \mathrm{h}^{-1}$.

6 Mean $\mathrm{HR}_{\max }$ and RPE at exhaustion were $196 \pm 6$ beats per min and $9.3 \pm 0.5$, respectively.

7

Distance covered and time to exhaustion

10 By design, distance covered during part A was the same in all three trials $(7.1 \pm 0.3 \mathrm{~km})$.

11 Time to exhaustion was significantly influenced by solution $\left(F_{2,12}=6.1, P<0.05, r=0.71\right)$, and was $34 \%$ greater in the MCHO trial compared with the HCHO trial $(5.5 \pm 0.8$ vs. $4.1 \pm$

$131.5 \mathrm{~min}, P<0.05, r=0.76)$, and by $14.6 \%$ compared with the LCHO trial $(4.8 \pm 1.2 \mathrm{~min})$, 14 although this was not statistically significant $(P=0.10, r=0.63)$. Time to exhaustion in the 15 LCHO trial was $17.1 \%$ greater than the $\mathrm{HCHO}$ trial, but was not statistically significant $(P=$ $160.09, r=0.63)$. Distance covered in part B was significantly greater in the MCHO trial 17 compared with the HCHO trial (931 \pm 172 vs. $706 \pm 272 \mathrm{~m}, P<0.05, r=0.76)$, but not the

18 LCHO trial $(811 \pm 230 \mathrm{~m}, P=0.09, r=0.63)$. Distance covered was not significantly

19 different between the LCHO and HCHO trials $(P=0.11, r=0.61)$. 
3 The mean time of all sprints, and the mean of participants' peak sprint time only, in each block of part A of the LIST are shown in figure 1A and 1B, respectively. There was a trend

5 for mean sprint times to be slower throughout exercise in the HCHO trial compared with the

6 other two trials, but no main effect of solution was present $\left(F_{2,10}=1.1, P=0.38, r=0.42\right)$.

7 Similarly, there was no interaction effect (solution $\mathrm{x}$ time, $F_{2.1,10.3}=0.89, P=0.44, r=0.39$ ).

8 There was a main effect of time $\left(F_{1.1,5.5}=8.6, P<0.05, r=0.79\right)$, with sprint time increasing

9 significantly with each successive exercise block $(P<0.05, r=0.56,0.82$ and 0.55,

10 respectively). Peak sprint time for each exercise block showed a trend for slower times in the 11 HCHO trial compared with the other two trials, but no main effect of solution $\left(F_{2,10}=1.1, P\right.$ $12=0.37, r=0.42)$ or interaction $\left(F_{6,30}=0.6, P=0.72, r=0.33\right)$ was found. There was a main effect of time $\left(F_{3,15}=8.3, P<0.005, r=0.79\right)$, with peak sprint time significantly slower in block 3 than block $2(P<0.001, r=0.75)$. There was no significant difference between blocks 1 and $2(P=0.22, r=0.30)$ or 3 and $4(P=0.60, r=0.10)$.

\section{PLEASE PLACE FIGURE 1A and 1B HERE}

Heart rate, ratings of perceived exertion, and gastric measures

21 Mean HR and RPE during part A of the LIST, and mean peak HR and RPE at exhaustion in 22 part B are shown in table 1. Heart rate was lower in the LCHO trial at all time points in part 23 A, but there was no significant treatment $\left(F_{2,8}=1.8, P=0.23, r=0.56\right)$ or interaction $\left(F_{6,24}\right.$ $24=1.7, P=0.62, r=0.40)$ effect. There was a main effect of time for $\mathrm{HR}$ in part A $\left(F_{3,12}=\right.$ 32.1, $P<0.001, r=0.94)$. Heart rate in block 2 was significantly greater than block $1(P<$ 
$10.01, r=0.98)$. There was no significant difference between blocks 2 and $3(P=0.48, r=$

$20.76)$ or 3 and $4(P=1.0, r=0.22)$. Peak HR at exhaustion in part B was not significantly

3 different between trials $\left(F_{1.1,6.8}=0.67, P=0.46, r=0.32\right)$. Ratings of perceived exertion

4 were similar at all time points between trials, with no significant differences found $\left(F_{2,12}=\right.$

$51.3, P=0.32, r=0.42)$. No interaction effect was present $\left(F_{6,36}=0.3, P=0.53, r=0.35\right)$.

6 There was a main effect of time $\left(F_{1.1,6.5}=36.0, P<0.005, r=0.93\right)$, with RPE significantly

7 greater in block 2 than block $1(P<0.05, r=0.97)$ and in block 4 than block $3(P<0.001, r$

$8=0.99)$. There was no significant difference between blocks 2 and $3(P=0.41, r=0.95)$, and

9 no significant between trials difference in RPE at exhaustion $\left(F_{1,6}=1.0, P=0.36, r=0.38\right)$.

PLEASE PLACE TABLE 1 HERE

Mean GF and GD during part A of the LIST and at exhaustion in part B are shown in table 2.

Mean GF was not significantly influenced by solution $\left(F_{2,12}=1.1, P=0.36, r=0.40\right)$, and there was no interaction effect $\left(F_{6,36}=1.0, P=0.43, r=0.38\right)$. There was a significant effect of time on $\mathrm{GF}\left(F_{3,18}=3.3, P<0.05, r=0.59\right)$. This main time effect was just under the stated alpha figure of $P<0.05$, and specific differences between time points could not be determined using post hoc analyses. Effect sizes for the differences between blocks 1 and 2, 2 and 3 , and 3 and 4 were $r=0.10,0.22$ and 0.48 , respectively. Gut fullness at exhaustion was not significantly different between trials $\left(F_{2,12}=2.2, P=0.16, r=0.51\right)$. Despite the significant main effect of time, GF scores during part A of the LIST were modest. There was no treatment $\left(F_{2,12}=0.4, P=0.68, r=0.25\right)$ or interaction $\left(F_{6,36}=1.8, P=0.14, r=0.48\right)$ effect on GD, but there was a main effect of time $\left(F_{3,18}=3.9, P<0.05, r=0.63\right)$. As with GF, specific differences could not be determined post hoc. Effect sizes for the differences between blocks 1 and 2, 2 and 3, and 3 and 4 were $r=0.46,0.10$ and 0.40 , respectively. 
1 Gastric discomfort at exhaustion was similar across all trials $\left(F_{2,12}=0.27, P=0.77, r=\right.$

2 0.21). Gastric discomfort scores during part A of the LIST were moderate.

3

6 Body mass loss and sweat rate

7

\section{Blinding}

\section{PLEASE PLACE TABLE 2 HERE}

Mean pre-exercise dry nude BM was not significantly different between trials $\left(F_{2,12}=0.1, P\right.$ $=0.92, r=0.11)$. Mean BM loss was $1.0 \pm 0.2,1.0 \pm 0.2$, and $1.0 \pm 0.4 \mathrm{~kg}$ in the LCHO, $\mathrm{MCHO}$ and $\mathrm{HCHO}$ trials, respectively $\left(F_{2,12}=0.11, P=0.90, r=0.13\right)$, equating to a mean loss of $1.62 \pm 0.37,1.63 \pm 0.24$, and $1.54 \pm 0.49 \%$ of pre-exercise $\mathrm{BM}$, respectively $\left(F_{2,12}=\right.$ $0.24, P=0.79, r=0.19)$. Mean SR was $0.78 \pm 0.15,0.78 \pm 0.13$, and $0.76 \pm 0.28{\mathrm{~L} . \mathrm{h}^{-1}}^{\text {in }}$ the LCHO, MCHO and HCHO trials, respectively $\left(F_{2,12}=0.03, P=0.97, r=0.07\right)$, equating to a BM-relative mean sweat loss of $12.63 \pm 2.81,12.53 \pm 1.75$, and $12.18 \pm 3.86 \mathrm{ml} \cdot \mathrm{kg}^{-1} \mathrm{BM} \cdot \mathrm{h}^{-1}$, respectively $\left(F_{2,12}=0.09, P=0.91, r=0.12\right)$.

After consuming the initial bolus of the solution immediately prior to exercise, one participant (14\%) correctly identified all solutions and six (86\%) failed to do so. Chi square analysis of the responses in the MCHO trial found a non-significant deviation from the expected response frequency $\left(\chi^{2}(1)=3.6, P=0.16\right)$. Post-exercise, only one participant correctly guessed all three solutions, and this was the same participant who guessed all three correctly pre-exercise. In the LCHO and MCHO trials, one participant (14\%) changed their mind post-exercise and correctly guessed the solution when they had guessed incorrectly 
1 prior to exercise. In the $\mathrm{HCHO}$ trial, no participant identified the correct solution postexercise after having chosen incorrectly pre-exercise.

Ambient temperature and relative humidity, fluid and carbohydrate intake

6 Mean ambient temperature and relative humidity during the LIST are shown in table 3. Mean ambient temperature was not significantly different between trials at any time point, therefore the data for all three trials was grouped for analysis of a main effect of time. Mean ambient temperature rose from $18.0 \pm 1.7^{\circ} \mathrm{C}$ immediately pre-exercise to $18.3 \pm 1.7^{\circ} \mathrm{C}$ at the end of part A of the LIST $\left(\chi^{2}(4)=39.3, P<0.001\right)$. Mean relative humidity was not significantly different between $\left(F_{2,12}=0.06, P=0.94, r=0.10\right)$ or within $\left(F_{1.6,9.6}=4.1, P=0.06, r=\right.$ $0.64)$ trials, and there was no interaction effect $\left(F_{2.2,2.4}=0.90, P=0.46, r=0.36\right)$.

Mean fluid intake was $811 \pm 83,810 \pm 82$, and $810 \pm 84 \mathrm{ml}\left(F_{2,12}=0.05, P=0.95, r=0.09\right)$ in the $\mathrm{LCHO}, \mathrm{MCHO}$ and $\mathrm{HCHO}$ trials, respectively. Absolute $\mathrm{CHO}$ intake in the LCHO, MCHO and HCHO trials was $12.7 \pm 1.3,37.6 \pm 3.7$, and $64.0 \pm 7.3$ g.h $^{-1}\left(F_{1.0,6.1}=497.0, P\right.$ $<0.001, r=0.99)$, or $0.21 \pm 0.02,0.63 \pm 0.06$, and $1.07 \pm 0.02 \mathrm{~g} \cdot \mathrm{min}^{-1}\left(F_{1.0,6.1}=481.6, P<\right.$

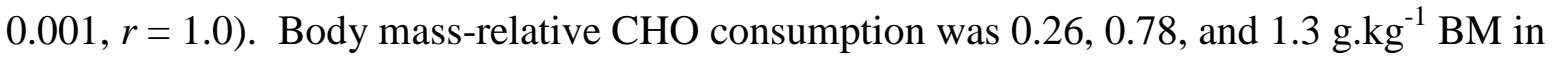
the $\mathrm{LCHO}, \mathrm{MCHO}$ and $\mathrm{HCHO}$ trials, respectively. 


\section{Discussion}

3 This study demonstrates that ingestion of a $6 \%$ CHO-E solution significantly improves the

4 intermittent endurance capacity of adolescent team games players during intermittent

5 endurance running compared with a $10 \%$ solution. A non-significant trend for improved

6 intermittent endurance capacity with the $6 \%$ compared with a $2 \%$ solution, and the $2 \%$

7 compared with a $10 \%$ solution, was also found. No significant influence of [CHO] was

8 found for sprint performance during the protocol.

10 Time to exhaustion

12 The greatest intermittent endurance capacity was achieved in the MCHO trial. This suggests

13 that adult guidelines for $\mathrm{CHO}$ ingestion during prolonged moderate- to high-intensity

14 exercise (Jeukendrup 2004) do not apply to adolescent team games athletes during

15 intermittent endurance running. In support of this adult-child difference, Nassis et al (1998)

16 failed to find a significant improvement in intermittent endurance capacity in adults during

17 prolonged intermittent treadmill running with ingestion of $\mathrm{CHO}$ at very similar rates to that of

18 the MCHO trial in the current study $\left(0.6 \mathrm{~g} \cdot \mathrm{min}^{-1} ; 34 \mathrm{~g} \cdot \mathrm{h}^{-1}\right)$.

20 The minimum absolute rate of $\mathrm{CHO}$ ingestion that has been demonstrated to enhance

21 endurance capacity during prolonged cycling in adults is $16 \mathrm{~g} \cdot \mathrm{h}^{-1}$ (Jeukendrup 2004), equating

22 to 0.26 g.min ${ }^{-1}$, whereas any further $\mathrm{CHO}$ ingestion above a rate of $\sim 1-1.1 \mathrm{~g} \cdot \mathrm{min}^{-1}$ does not

23 further increase the rate of $\mathrm{CHO}_{\text {exo }}$ oxidation, provided the composition of ingested $\mathrm{CHO}$

24 remains the same (Jeukendrup 2004). In the current study, the rate of CHO ingestion in the

25 LCHO trial may not have facilitated absorption of sufficient $\mathrm{CHO}$ into the systemic 
1 circulation to enable exercise enhancement (Rogers et al 2005) The reasons behind the

2 inferior intermittent endurance capacity in the HCHO trial are unclear. It does not appear that

3 CHO ingestion simply exceeded the maximal rate of $\mathrm{CHO}$ absorption and/or oxidation of the

4 participants, as in this case a similar intermittent endurance capacity between the HCHO and

5 MCHO trials would have been expected. This requires further study, perhaps by focussing

6 initially on potential modulators of $\mathrm{CHO}_{\text {exo }}$ oxidation (Jeukendrup 2004; Shi \& Passe 2010).

7 However, it should also be noted that there was a larger variation in time to exhaustion values

8 for the $\mathrm{HCHO}$ trial, perhaps representing a greater individual variation in response to this

9 CHO dose. Conversely, this may also have been due to the relatively small sample size.

10 While this study was not designed to identify enhancement mechanisms due to ethical and

11 consensual restrictions, it does provide initial indirect support for the existence of low and

12 high $\mathrm{CHO}$ ingestion thresholds during intermittent endurance running in adolescents, below

13 and above which the efficacy of CHO does not appear to be maximised, in line with adult

14 findings (Jeukendrup 2004). Interestingly, these thresholds appear to be at different ingestion

15 rates for adolescents than adults. Clearly, more work is required to confirm the relative

16 influence of different [CHO] during intermittent endurance running in adolescents, and to

17 provide data on the metabolic response to these [CHO], which may help to explain the

18 performance data.

Sprint Performance

22 The lack of influence of $\mathrm{CHO}$ on sprint performance, along with a progressive, treatment23 independent increase in sprint duration with time, in the current study is in line with previous 24 work from this laboratory (Phillips et al 2010), and the reader is referred here for further 25 discussion on these findings. When the results of these two studies are combined, it can be 
1 inferred that $\mathrm{CHO}$ supplementation across a range of ingestion rates does not significantly

2 influence the sprint performance of adolescent team games players during intermittent

3 endurance running, in line with most adult work (Davis et al, 1999; Nicholas et al., 1995).

5 The mean increase in sprint time from the first to the last block of part A in all trials is greater

6 than that recorded in some adult work (0.08 sec for both trials; Ali et al 2007), in line with

7 Phillips et al (2010). This provides further confirmation that adolescent team games players

8 do not appear to display a greater fatigue resistance than adults during sprinting in the LIST,

9 as may have been expected (Ratel et al 2002).

10

11 Heart rate, ratings of perceived exertion, and gastric measures

13 The lack of a treatment effect on HR during part A of the LIST in the current study agrees

14 with our previous findings (Phillips et al 2010) and most adult research (Ali et al 2007;

15 Nicholas et al 1995; Welsh et al 2002). Mean HR during part A of the LIST was in

16 agreement with values reported during outdoor 11-a-side and indoor 5-a-side soccer matches

17 in recreational and elite young players (Castagna et al., 2007; Strøyer et al., 2004).

19 Heart rate at exhaustion in part B of the current study is in contrast to our previous

20 investigation, which reported a significantly greater $\mathrm{HR}$ at exhaustion in the CHO trial

21 (Phillips et al 2010). It is possible that this was simply an artefact of the particular participant

22 population used in that study and not, as suggested at the time, a mechanistic indicator of a

23 metabolic and/or perceptual response to $\mathrm{CHO}$ supplementation. Alternatively, a perceptual

24 mechanism of $\mathrm{CHO}$ efficacy in adolescent team games players may exist, but may be

25 participant-dependent. More work should be undertaken to clarify this. 
2 Data from the current study lends further support to our previous finding that $\mathrm{CHO}$

3 supplementation does not modulate RPE during intermittent endurance running in

4 adolescents (Phillips et al 2010), although it should be considered that a non-CHO trial was

5 not included in the current study. This suggests that $\mathrm{CHO}$ supplementation does not elicit

6 centrally-mediated alterations in adolescents that modulate effort perception during exercise,

7 and may further indicate that enhancements in intermittent endurance capacity with $\mathrm{CHO}$

8 ingestion in this population are of a metabolic nature. However, as mentioned in the previous

9 paragraph, it may be too early to rule out a possible influence of $\mathrm{CHO}$ on effort perception at 10 exhaustion.

12 No increase in RPE was observed in block 3 of part A of the LIST. Both mean sprint and 13 mean peak sprint times significantly slowed during block 3 of the LIST, which may have had 14 an attenuating influence on RPE in block 3. However, a slower sprint time may relate to a 15 less favourable metabolic condition for sprinting (Bangsbo et al 2006), not necessarily a 16 reduced effort from participants. Furthermore, in our previous study mean sprint and mean 17 peak sprint time slowed significantly in block 3 of the LIST, with no corresponding 18 attenuation in RPE (Phillips et al 2010). The non-significant increase in RPE in block 3 of 19 the LIST in the current study may be a result of the low participant number, which is 20 supported by the almost perfect ES for this time point.

22 The current study indicates that 2, 6, and 10\% CHO-E solutions are equally well tolerated by 23 adolescents during intermittent endurance running. This conforms to previous findings from 24 this laboratory regarding tolerance to $\mathrm{CHO}-\mathrm{E}$ solutions and $\mathrm{CHO}$ gels in adolescents during 25 prolonged intermittent exercise (Phillips et al 2010; Phillips et al unpublished data). 
1 Mechanisms behind the influence of time on gastric variables are discussed elsewhere

2 (Phillips et al 2010).

$4 \quad$ Body mass loss and sweat rate

6 When the results of the current, and our previous, study are considered, it appears that

7 ingestion of $\mathrm{CHO}$ across a range of concentrations does not alter the BM loss or SR responses

8 of adolescents to intermittent endurance running. The BM loss and SR data from the current

9 study and that of Phillips et al (2010) currently represent the only published data of its kind in

10 adolescents during intermittent endurance running, therefore comparison of values with other

11 related work is not possible at this time.

\section{Blinding}

15 It appears that the blinding procedures used in this study were effective. Furthermore, the

16 data indicates that exercise did not provide any cues enabling participants to more accurately

17 identify the three solutions. Interestingly, however, it does appear that some cues were

18 provided to the participants during exercise that led them to falsely believe that they had not

19 received the $\mathrm{HCHO}$ solution. It is impossible to speculate as to what these cues may have

20 been, and whether they were perceived as positive or negative, without knowing what the

21 individual participants' beliefs were pre-exercise regarding the $\mathrm{HCHO}$ solution. Therefore,

22 the influence of intermittent endurance running exercise on adolescents' perceptions of $\mathrm{CHO}$

23 administration should be investigated further, considering the potential influence of an

24 individuals perception of the treatment they believe they have received on their subsequent

25 exercise performance (Beedie et al 2007). 
Preliminary tests: peak running velocity and maximum heart rate

4 The mean $\mathrm{V}_{\text {peak }}$ of $14.4 \mathrm{~km} \cdot \mathrm{h}^{-1}$ in the current study is very similar to that reported in our 5 previous work (Phillips et al 2010), and suggests our participants were of a notably higher

6 training status than international population means (Sandercock et al 2008), although protocol

7 differences should be considered when interpreting $\mathrm{V}_{\text {peak }}$ data between studies. The mean

$8 \mathrm{HR}_{\max }$ and RPE values recorded in the current study are again similar to our previous work,

9 and indicate our participants provided a maximal effort during the incremental test

10 (Armstrong, 2007). The similar $\mathrm{V}_{\text {peak }}, \mathrm{HR}$ and RPE data in the current study compared with

11 our previous work (Phillips et al 2010) indicates that the participants used in these two

12 studies were of a similar training status, strengthening the comparisons made throughout this

13 discussion.

\section{Conclusion}

17 Ingestion of a $6 \%$ CHO-E solution significantly improves the intermittent endurance capacity of adolescent team games players during intermittent endurance running compared with a $10 \%$ solution. A non-significant trend for greater intermittent endurance capacity was

20 reported with ingestion of the $6 \%$ compared with the $2 \%$ solution, and the $2 \%$ compared with

21 the $10 \%$ solution. Carbohydrate concentration did not significantly influence sprint

22 performance or physiological responses to intermittent endurance running. Future research

23 should build on these findings in order to further develop guidelines for optimal $\mathrm{CHO}$

24 ingestion by adolescents during team games. 


\section{Acknowledgements}

2

3 The authors gratefully acknowledge the support of High5 Ltd, Bardon, Leicestershire, UK for

4 the supply of maltodextrin, electrolyte tablets, and drink bottles to enable completion of this

5 study. We wish to extend a special thanks to the staff, pupils and parents of George Watsons

6 College, Edinburgh, for their invaluable participation in, and support of, this research project.

7

8

9

10

11

12

13

14

15

16

17

18

19

20

21

22

23

24

25 


\section{$1 \quad$ Ethical Declaration}

2

3 The authors confirm that the conduct of this study complied fully with current Scottish law,

4 and with the full ethical approval of the University of Edinburgh, Moray House School of

$5 \quad$ Education Ethics Committee.

6

7

8

9

10

11

12

13

14

15

16

17

18

19

20

21

22

23

24

25 


\section{Reference List}

2

3 Ali A, Williams C (2009) Carbohydrate ingestion and soccer skill performance during

4 prolonged intermittent exercise. J Sports Sci 27: 1499-1508.

5

6 Ali A, Williams C, Nicholas W, Foskett A (2007) The influence of carbohydrate-electrolyte

$7 \quad$ ingestion on soccer skill performance. Med Sci Sports Exerc 39: 1969-1976.

8

9 Armstrong N (Ed) (2007) Paediatric Exercise Physiology. Churchill Livingstone Elsevier, 10 Philadelphia.

11

12 Aucouturier J, Baker JS, Duché P (2008) Fat and carbohydrate metabolism during

13 submaximal exercise in children. Sports Med 38: 213-238.

14

15 Bangsbo J (1994) The physiology of soccer with special reference to intense intermittent 16 exercise. Acta Physiol Scand (Suppl) 619: 1-155.

17

18 Bangsbo J, Mohr M, Krustrup P (2006) Physical and metabolic demands of training and 19 match-play in the elite football player. J Sports Sci 24: 665-674.

21 Baxter-Jones ADG, Eisenmann JC, Sherer LB (2005) Controlling for maturation in pediatric exercise science. Pediatr Exerc Sci 17: 18-30.

23 Beedie CJ, Coleman DA, Foad AJ (2007) Positive and negative placebo effects resulting

24 from the deceptive administration of an ergogenic aid. Int J Sport Nutr Exerc Metab 17: 25925269. 
1 Castagna C, Belardinelli R, Impellizzeri FM, Abt GA, Coutts AJ, D’Ottavio S (2007)

2 Cardiovascular responses during recreational 5-a-side indoor-soccer. J Sci Med Sport 10: 3 89-95.

4

5 Christmass MA, Dawson B, Passeretto P, Arthur PG (1999) A comparison of skeletal muscle 6 oxygenation and fuel use in sustained continuous and intermittent exercise. Eur J Appl

$7 \quad$ Physiol 80: 423-435.

8

9 Clarke ND, Drust B, Maclaren DPM, Reilly T (2008) Fluid provision and metabolic

10 responses to soccer-specific exercise. Eur J Appl Physiol 104: 1069-1077.

12 Crouter S, Foster C, Esten P, Brice G, Porcari JP (2001) Comparison of incremental treadmill 13 exercise and free range running. Med Sci Sports Exerc 33: 644-647.

Davis JM, Welsh RS, De Volve KL, Alderson NA (1999) Effects of branched-chain amino acids and carbohydrate on fatigue during intermittent, high-intensity running. Int J Sports

17 Med 20: 309-314.

Edwards AM, Mann ME, Marfell-Jones MJ, Rankin DM, Noakes TD, Shillington DP (2007)

20 Influence of moderate dehydration on soccer performance: physiological responses to $45 \mathrm{~min}$

21 of outdoor match-play and the immediate subsequent performance of sport-specific and

22 mental concentration tests. Br J Sports Med 41: 385-391.

24 Ekblom B (1986) Applied physiology of soccer. Sports Med 3: 50-60. 
1 Field A (2005) Discovering Statistics Using SPSS (2 ${ }^{\text {nd }}$ ed). Sage, London.

2

3 Hopkins WG (2006) A Scale of Magnitudes for Effect Statistics. Sportscience.

4 http://sportsci.org/resource/stats/. Accessed 19 January 2011.

5

6 Jeukendrup A (2004) Carbohydrate intake during exercise and performance. Nutrition 20:

$7 \quad 669-677$.

8

9 Jeukendrup AE, Wagenmakers AJM, Stegen JHCH, Gijsen AP, Brouns F \& Saris WHM

10 (1999) Carbohydrate ingestion can completely suppress endogenous glucose production

11 during exercise. J Physiol 276: E672-E683.

12

13 Lavcanska V, Taylor NF \& Schache AG (2005) Familiarization to treadmill running in young

14 unimpaired adults. Hum Mov Sci 24: 544-557.

15

16 Marino FE, Lambert MI, Noakes TD (2004) Superior performance of African runners in

17 warm humid but not in cool environmental conditions. J Appl Physiol 96: 124-130.

18

19 Mirwald RL, Baxter-Jones ADG, Bailey DA, Beunen GP (2002) An assessment of maturity

20 from anthropometric measurements. Med Sci Sports Exerc 34: 689-694.

21

22 Murray R, Eddy DE, Murray TW, Seifert JG, Paul GL, Halaby GA (1987) The effect of fluid and carbohydrate feedings during intermittent cycling exercise. Med Sci Sports Exerc 19:

24 597-604. 
1 Nassis GP, Williams C, Chisnall P (1998) Effect of a carbohydrate-electrolyte drink on

2 endurance capacity during prolonged intermittent high-intensity running. Br J Sports Med

$3 \quad 32: 248-252$.

4

5 Nicholas CW, Williams C, Lakomy HKA, Phillips G, Nowitz A (1995) Influence of

6 ingesting a carbohydrate-electrolyte solution on endurance capacity during intermittent, high-

7 intensity shuttle running. J Sports Sci 13: 283-290.

8

9 Phillips SM, Turner AP, Gray S, Sanderson MF, Sproule J (2010) Ingesting a 6\%

10 carbohydrate-electrolyte solution improves endurance capacity, but not sprint performance,

11 during intermittent, high-intensity shuttle running in adolescent team games players aged 12-

1214 years. Eur J Appl Physiol 109: 811-821.

13

14 Ratel S, Bedu M, Hennegrave A, Doré E, Duché P (2002) Effects of age and recovery

15 duration on peak power output during repeated cycling sprints. Int J Sports Med 23: 397-

16402.

17

18 Riddell MC (2008) The endocrine response and substrate utilization during exercise in

19 children and adolescents. J Appl Physiol 105: 725-733.

20

21 Roemmich JN, Barkley JE, Epstein LH, Lobarinas CL, White TM, Foster JH (2006) Validity of PCERT and OMNI walk/run ratings of perceived exertion. Med Sci Sports Exerc 38: 1014-1019. 
1 Rogers J, Summers RW, Lambert GP (2005) Gastric emptying and intestinal absorption of a

2 low-carbohydrate sport drink during exercise. Int J Sport Nutr Exerc Metab 15: 220-235.

3

4 Rosnow RL, Rosenthal R (2005) Beginning Behavioural Research: A Conceptual Primer (5 ${ }^{\text {th }}$ 5 ed). Pearson/Prentice Hall, New Jersey.

6

7 Sandercock G, Voss C, Gladwell V (2008) Twenty-metre shuttle run test performance of

8 English children aged $11-15$ years in 2007: comparisons with international standards. J

$9 \quad$ Sports Sci 26: 953-957.

10

11 Shi X, Horn MK, Osterberg KL, Stofan JR, Zachwieja JJ, Horswill CA, Passe DH, Murray R 12 (2004) Gastrointestinal discomfort during intermittent high-intensity exercise: effect of 13 carbohydrate-electrolyte beverage. Int J Sport Nutr Exerc Metab 14: 673-683.

Shi X, Passe DH (2010) Water and solute absorption from carbohydrate-electrolyte solutions 16 in the human proximal small intestine: A review and statistical analysis. Int J Sport Nut 17 Exerc Metab 20: 427-442.

Strøyer J, Hansen L, Klausen K (2004) Physiological profile and activity pattern of young 20 soccer players during match play. Med Sci Sports Exerc 36: 168-174.

22 Tanner JM (1962) Growth at Adolescence. Oxford: Blackwell Scientific Publications. 23

24 Timmons BW, Bar-or O, Riddell MC (2003) Oxidation rate of exogenous carbohydrate 25 during exercise is higher in boys than in men. J Appl Physiol 94: 278-284. 
1 Timmons BW, Bar-Or O, Riddell MC (2007) Influence of age and pubertal status on

2 substrate utilization during exercise with and without carbohydrate intake in healthy boys.

3 Appl Physiol Nutr Metab 32: 416-425.

4

5 van Nieuwenhoven MA, Brouns F, Kovacs EMR (2005) The effect of two sports drinks and

6 water on GI complaints and performance during an 18-km run. Int J Sports Med 26: 281-

7285.

8

9 Welsh RS, Davis JM, Burke JR, Williams HG (2002) Carbohydrates and physical/mental 10 performance during intermittent exercise to fatigue. Med Sci Sports Exerc 34: 723-731. 11 


\section{$1 \quad$ Tables}

3 Table 1 Mean heart rate (beats per min) and mean ratings of perceived exertion during part

4 A of the LIST, and peak heart rate and ratings of perceived exertion at exhaustion in part B 5 for all trials.

\section{Period of the LIST}

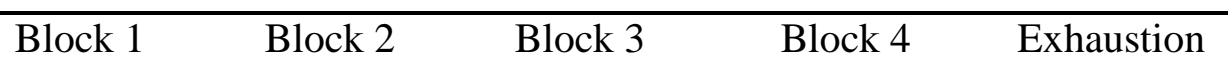

\section{Mean heart rate}

(beats per min)

$\begin{array}{lccccc}\text { LCHO } & 159 \pm 7 & 164 \pm 7^{* * *} & 165 \pm 7 & 165 \pm 7 & 189 \pm 3 \\ \text { MCHO } & 162 \pm 6 & 168 \pm 5^{* * *} & 170 \pm 6 & 169 \pm 5 & 190 \pm 4 \\ \text { HCHO } & 162 \pm 5 & 168 \pm 6^{* * *} & 168 \pm 6 & 168 \pm 5 & 190 \pm 4\end{array}$

\section{Mean ratings of} perceived exertion

$\begin{array}{llllll}\text { LCHO } & 4.6 \pm 1.1 & 6.0 \pm 0.82^{*} & 7.4 \pm 0.98 & 7.6 \pm 1.1 * * & 9.4 \pm 0.53 \\ \text { MCHO } & 4.4 \pm 0.98 & 6.1 \pm 0.70^{*} & 6.7 \pm 0.95 & 8.0 \pm 0.82 * * & 9.3 \pm 0.49 \\ \text { HCHO } & 4.4 \pm 0.98 & 6.0 \pm 1.0 * & 7.1 \pm 0.70 & 8.0 \pm 0.82 * * & 9.3 \pm 0.49\end{array}$

$6 \quad$ Data are mean $\pm \mathrm{SD}(n=7)$

$7 \mathrm{LCHO}=$ low $\mathrm{CHO}$ trial; $\mathrm{MCHO}=$ moderate $\mathrm{CHO}$ trial; $\mathrm{HCHO}=$ high $\mathrm{CHO}$ trial

$8 * * *$ significantly greater than block $1, P<0.01$; * significantly greater than previous block, $9 \quad P<0.05 ; * *$ significantly greater than previous block, $P<0.001$ 
1 Table 2 Mean gut fullness and gastric discomfort ratings during part A of the LIST, and at 2 exhaustion in part B, for all trials.

Period of the LIST

\begin{tabular}{|c|c|}
\hline Block 1 & Block 2 \\
\hline
\end{tabular}

\section{Mean gut fullness}

ratings

$\begin{array}{llllll}\text { LCHO } & 4.1 \pm 1.7 & 4.7 \pm 1.3 & 4.7 \pm 1.1 & 5.3 \pm 1.3 & 5.1 \pm 1.1 \\ \text { MCHO } & 4.1 \pm 1.6 & 3.9 \pm 1.9 & 4.0 \pm 1.7 & 4.7 \pm 1.7 & 5.3 \pm 1.3 \\ \text { HCHO } & 3.7 \pm 1.4 & 3.7 \pm 1.1 & 4.3 \pm 1.6 & 4.3 \pm 1.1 & 4.7 \pm 0.80\end{array}$

Mean gastric

discomfort ratings

$\begin{array}{llllll}\text { LCHO } & 2.1 \pm 1.1 & 3.1 \pm 1.2 & 3.0 \pm 1.3 & 3.1 \pm 1.5 & 4.0 \pm 1.5 \\ \text { MCHO } & 2.9 \pm 1.3 & 3.3 \pm 1.4 & 3.3 \pm 1.6 & 3.4 \pm 1.7 & 4.3 \pm 1.8 \\ \text { HCHO } & 2.3 \pm 1.9 & 2.4 \pm 1.1 & 2.9 \pm 1.2 & 4.0 \pm 1.9 & 4.1 \pm 2.0\end{array}$

3 Data are mean $\pm \operatorname{SD}(n=7)$

$4 \mathrm{LCHO}=$ low $\mathrm{CHO}$ trial; $\mathrm{MCHO}=$ moderate $\mathrm{CHO}$ trial; $\mathrm{HCHO}=$ high $\mathrm{CHO}$ trial

5 A main effect of time was found for GF and GD $(P<0.05)$ 
1 Table 3 Mean ambient temperature $\left({ }^{\circ} \mathrm{C}\right)$ and relative humidity $(\%)$ immediately before, and 2 during, part A of the LIST.

Period of the LIST

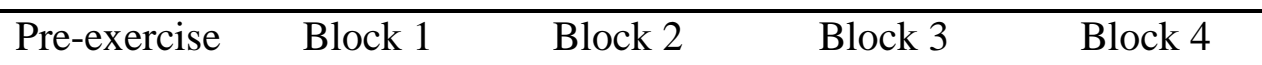

\section{Mean ambient}

temperature $\left({ }^{\circ} \mathrm{C}\right)$

$\begin{array}{lrrrrr}\text { LCHO } & 18.0 \pm 1.8 & 18.1 \pm 1.9 & 18.1 \pm 1.9 & 18.2 \pm 1.9 & 18.2 \pm 1.9 \\ \text { MCHO } & 17.8 \pm 1.4 & 17.9 \pm 1.4 & 18.0 \pm 1.4 & 18.0 \pm 1.3 & 18.1 \pm 1.3 \\ \text { HCHO } & 18.3 \pm 2.2 & 18.4 \pm 2.1 & 18.4 \pm 2.1 & 18.5 \pm 2.1 & 18.5 \pm 2.1\end{array}$

Mean relative

humidity (\%)

$\begin{array}{llllll}\text { LCHO } & 40.6 \pm 5.5 & 40.1 \pm 6.1 & 39.9 \pm 6.1 & 39.4 \pm 5.9 & 39.0 \pm 6.0 \\ \text { MCHO } & 40.7 \pm 8.4 & 40.4 \pm 8.4 & 40.1 \pm 8.6 & 39.7 \pm 8.6 & 39.6 \pm 9.2 \\ \text { HCHO } & 40.6 \pm 9.0 & 41.0 \pm 8.3 & 41.1 \pm 8.2 & 41.0 \pm 8.3 & 40.0 \pm 8.8\end{array}$

3 Data are mean $\pm \operatorname{SD}(n=7)$

$4 \mathrm{LCHO}=$ low $\mathrm{CHO}$ trial; $\mathrm{MCHO}=$ moderate $\mathrm{CHO}$ trial; $\mathrm{HCHO}=$ high $\mathrm{CHO}$ trial

5 A main effect of time was found for the grouped mean ambient temperature data $(P<0.01)$ 
a

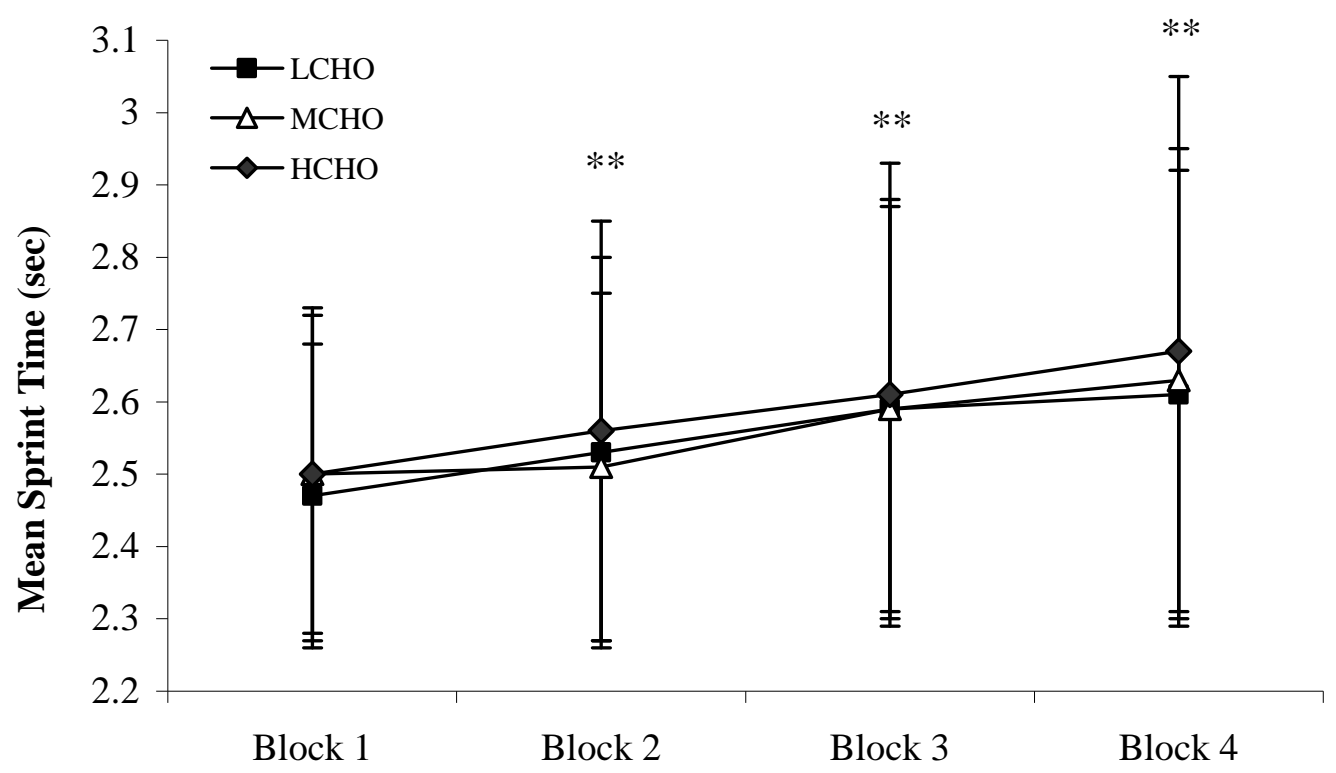

\section{Period of LIST}

1

b

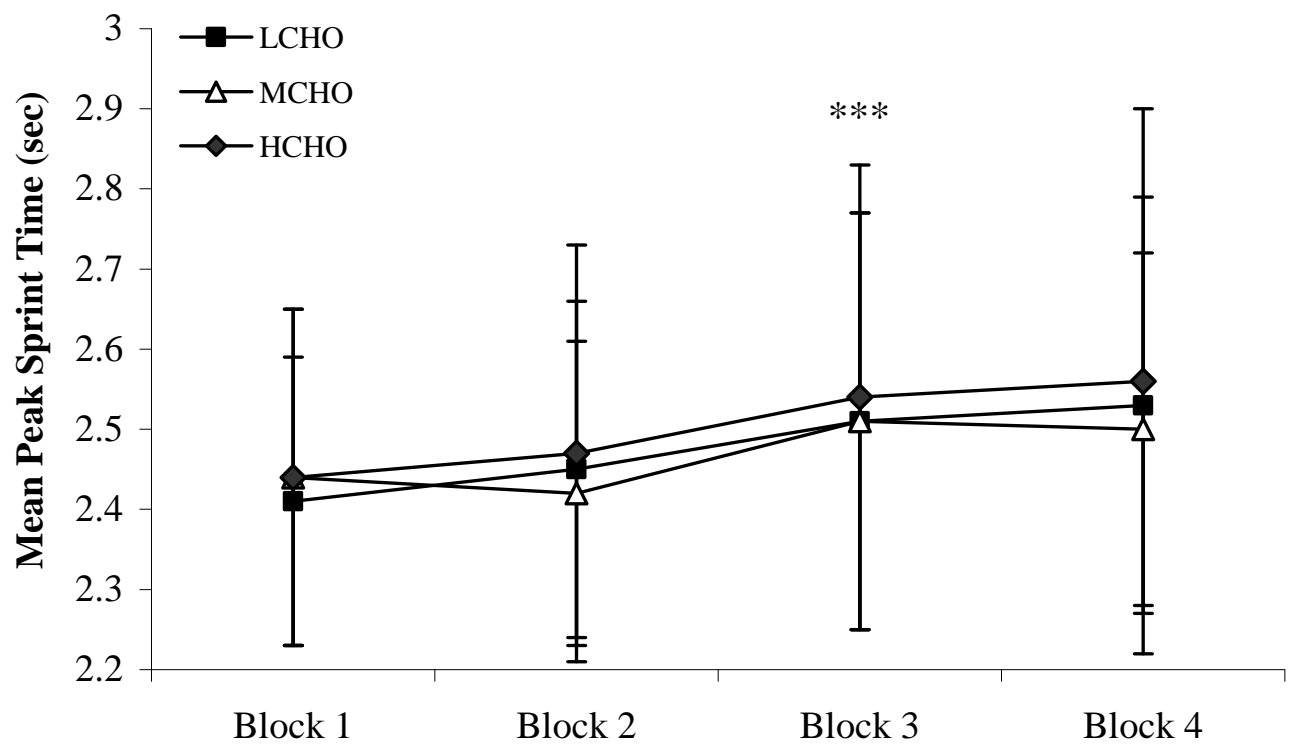

Period of LIST

2

4 Figure 1 Mean sprint time (A) and mean peak sprint time (B) during part A of the LIST for

5 allboth trials. * significantly greater than previous block, $P<0.05$; $* *$ significantly greater

6 than previous block, $P<0.001 .(n=6)$. 